\title{
Industrielle Bildwelten im späten Zarenreich - Einleitung
}

Im ausgehenden 19. Jahrhundert veränderten sich die Silhouetten russischer Städte. Hatten bisher besonders Türme und goldene Kuppeln orthodoxer Kirchen die Aufmerksamkeit von Einheimischen und Reisenden auf sich gezogen, bekamen sie jetzt Konkurrenz von profanen Bauwerken der industriellen Entwicklung des späten Zarenreichs. Fabrikbauten entwickelten sich zum festen Bestandteil urbaner Zentren und Gewerbedörfern. Hohe Fassaden und kastenartige Baukörper überragten kleinere Sakralbauten und ihre rauchenden Schornsteine dominierten um die Jahrhundertwende vielerorts die Stadtbilder. ${ }^{1}$ Die neuen Gebäude aus Backstein, Glas und Stahl waren Manifestationen eines gesellschaftlichen Wandels, der gerade in den Städten des Zarenreichs unübersehbare Spuren hinterlassen hatte. Dokumentiert wurden diese Veränderungen im Russischen Reich mit dem damals fortschrittlichsten Bildmedium, der Fotografie. ${ }^{2}$

Die neuen Bilder schienen wie gemacht für Abbildungen von Maschinen und Fabrikanlagen, waren sie doch selbst das Ergebnis eines technischen Verfahrens. ${ }^{3}$ Der menschliche Einfluss auf die Bildgestaltung schien marginal zu sein, allein das chemische Verfahren ließ die neuen Aufnahmen entstehen, so eine weit verbreitete Annahme. Beim Ersten Kongress der russischen Vertreter der Photographie äußerte ein Teilnehmer: „Die Photographie erfordert keine besonderen Talente, und für die gesamte Lichtbildkunst ist der einzige

1 Karl Schlögel: Jenseits des Großen Oktober. Das Laboratorium der Moderne, Petersburg 1909-1921, Berlin 1988, S. 30-49; vergleichsweise zu anderen Regionen Europas: Friedrich Lenger: Metropolen der Moderne. Eine europäische Stadtgeschichte seit 1850, München 2013, S. 51-54, 179-188; Dorothy and Alan Shelston: The Industrial City, 1820-1870, Basingstoke 1990, S. 1-14; Detlev Vonde: Revier der großen Dörfer. Industrialisierung und Stadtentwicklung im Ruhrgebiet, Hagen 1989.

2 Bernd Stiegler: Philologie des Auges. Die photographische Entdeckung der Welt im 19. Jahrhundert, München 2001; Jonathan Crary: Techniken des Betrachters. Sehen und Moderne im 19. Jahrhundert, Dresden 1996; Wilfried Weigand (Hrsg.): Frühzeit der Photographie. 1826189o, Frankfurt a. M. 1980; Jürgen Osterhammel: Die Verwandlung der Welt. Eine Geschichte des 19. Jahrhunderts, München 2009, S. 76-80.

3 Gerhard Paul: Das visuelle Zeitalter. Punkt und Pixel, Göttingen 2016, S. 23-26. 
Künstler die Sonne. ${ }^{4}$ Diese Ansicht rief in der zweiten Hälfte des 19. Jahrhunderts unter Fotografen ${ }^{5}$ und Künstlern Diskussionen darüber hervor, ob es sich bei Fotografen um Künstler handle oder um gewöhnliche Handwerker. ${ }^{6}$ Die fotografischen Aufnahmen gäben nur eine Masse an Details wieder. Sie seien jedoch nicht in der Lage, die Atmosphäre eines Orts oder den Charakter eines Menschen einzufangen, so die Kritiker. Gerade diese Detailgenauigkeit machte die Fotografien jedoch für Industrielle ${ }^{7}$ interessant, denn in den Werkshallen ihrer Unternehmen reihte sich eine unübersehbare Zahl von kleinen und großen Maschinen aneinander. Maler und Grafiker waren kaum in der Lage, dieses unübersichtliche Gewirr aus Treibriemen und Rädern, Zylindern und Achsen überzeugend wiederzugeben. Das neue visuelle Medium der Fotografie bildete die industriellen Anlagen hingegen scheinbar mühelos ab. ${ }^{8}$ Gleichzeitig waren Fotografien aufgrund des von Henry Fox Talbot (1800-1877)

4 Zitiert nach: Elena Valentinovna Barchatova: Wissenschaft? Handwerk? Kunst!, in: Nikolaj Nikolaevič Rachmanov (Hrsg.): Das Russland der Zaren. Photographien von 1839 bis zur Oktoberrevolution, Berlin 1989, S. 7-36, S. 27. Diese Diskussion führten Intellektuelle in ganz Europa. Zu den Standpunkten prominenter Wortführer ein Überblick bei: Peter Geimer: Theorien der Fotografie. Zur Einführung, Hamburg 2009, S. 170-191.

5 Männliche Formulierungen schließen, wenn nicht speziell gekennzeichnet, die weibliche Form mit ein.

6 David Elliot: The Photograph in Russia. Icon of a New Age, in: ders. (Hrsg.): Photography in Russia 1840-1940, London 1992, S. 11-22, S. 12; Elena Valentinovna Barchatova: Pictoralism. Photography as Art, in: David Elliott (Hrsg.): Russische Photographie 1840-1940, Berlin 1993, S. 51-6o, S. 51, 54; Valerij Timofeevič Stigneev: Vek fotografii, 1894-1994, Bd. 1. Očerki istorii otečestvennoj fotografii, Moskva 2007, S. 19, 21; Jens Jäger: Gesellschaft und Photographie. Formen und Funktionen der Photographie in Deutschland und England 1839-1860, Opladen 1996, S. 141; Céline Assegond: La photographie du travail. Chantiers, usines et mines (18501915), Analyse des modalités de représentation, Mémoire de recherche approfondie (3e cycle), Histoire de la photographie, Soutenu le 25 juin 2012, École du Louvre, S. 45.

7 In der vorliegenden Arbeit ist immer wieder von „Industriellen“, „Fabrikbesitzern“ oder „Unternehmern“ die Rede. Mit Blick auf die Verwendung von Fotografien beziehen sich die entsprechenden Aussagen auf diejenigen Akteure, von deren Fabriken Fotografien überliefert sind; Handwerksbetriebe und kleine Fabriken werden daher ausgeklammert. Möglicherweise könnte es lohnenswert sein, in weiterführenden Studien unterschiedliche Gruppen von Unternehmern genauer zu untersuchen.

8 Timm Starl bezeichnet die Konzentration auf Details als Charakteristik einer neuen Sichtweise, die sich in den $1890 e r$ Jahren in Europa ausbildete. Timm Starl: Im Prisma des Fortschritts. Zur Fotografie des 19. Jahrhunderts, Marburg 1991, S. 93. 
entwickelten Negativ-Positiv-Verfahrens reproduzierbar und ließen sich in großer Zahl herstellen: ${ }^{9}$ sie waren somit selbst Teil der Industrialisierung. ${ }^{10}$

Im heutigen Zeitalter der Smartphones sind Fotografien allgegenwärtig und verbreiteter denn je. Neben privaten Schnappschüssen oder Pressebildern konsumieren wir täglich unzählige Werbefotografien von Unternehmen. Die vorliegende Arbeit widmet sich einer Frühform dieser Fotografie. Neben Reklameaufnahmen wird der gesamte Bereich des Zusammenspiels der damals neuen Bilder und der russischen Industrie im langen 19. Jahrhundert in den Blick genommen. Es wird untersucht, auf welche Weise die Fotografie Betriebe im Zarenreich beeinflusste und wie Akteure der Industrialisierung die neuen Bilder für ihre Interessen einsetzten. In der Arbeit wird weiter die Darstellung von Fabriken analysiert und gefragt, wie sich der Blick durch die Kamera auf Industrieanlagen bis zur Oktoberrevolution 1917 veränderte. Quellengrundlage hierfür sind einzelne Fotografien und Abzüge, Alben sowie Festschriften aus Firmenarchiven, illustrierte Zeitschriften und Postkarten. Die Arbeit fragt, welchen Blick die Bildwelten des Zarenreichs auf Fabriken und Industrieanlagen warfen und wie er sich je nach Medium unterschied.

Bildquellen spielen gerade für die Geschichte des Zarenreichs eine wichtige Rolle, weil große Teile der Bevölkerung weder lesen noch schreiben konnten. Insbesondere in ländlichen Gebieten waren noch in den 1910er Jahren knapp drei Viertel der russischen Bevölkerung Analphabeten. ${ }^{11}$ Was die Betrachter der Fotografien genau sahen und welchen Eindruck die Bilder bei ihnen hinterließen, ist schwer zu sagen, denn es sind keine russischen Memoiren bekannt, deren Autoren sich zu Fabrikfotografien äußern. ${ }^{12}$ Auch Monica Rüthers weist

$9 \quad$ Zur Reproduzierbarkeit der Fotografie: Walter Benjamin: Das Kunstwerk im Zeitalter seiner technischen Reproduzierbarkeit, in: Rolf Tiedemann; Hermann Schweppenhäuser (Hrsg.): Walter Benjamin: Gesammelte Schriften, Bd. I.2, Frankfurt a. M. 1974, S. 435-508, S. $441-446$.

10 Reinhard Matz: Industriefotografie. Aus Firmenarchiven des Ruhrgebiets, Essen 1987, S. 13 .

11 In den urbanen Zentren konnten deutlich mehr Menschen lesen und schreiben, um die Jahrhundertwende konnten ca. 7o Prozent der männlichen Bevölkerung lesen. Jeffrey Brooks: When Russia Learned to Read. Literacy and Popular Literature, 1861-1917, Princeton 1985, S. 4.

12 Auseinandersetzungen und Diskussionen über die Standpunktgebundenheit und Relativität historischer Realitäten sind schon lange Teil der Geschichtswissenschaft. Zu dieser Tradition beispielsweise: Dieter Langewiesche: Erinnerungsgeschichte und Geschichtsnormierung, in:Niklaus Buschmann; Ute Planert (Hrsg.); Dieter Langewiesche:Zeitwende. Geschichtsdenken heute, Göttingen 2008, S. 21-40, S. 31; Otto Gerhard Oexle: „Wirklichkeit“ - „Krise der Wirklichkeit“ - „Neue Wirklichkeit“. Deutungsmuster und Paradigmenkämpfe in der deutschen Wissenschaft vor und nach 1933, in: Andrea von Hülsen-Esch; Bernhard Jussen; Frank Rexroth (Hrsg.); Otto Gerhard Oexle: Die Wirklichkeit und das 
auf die Schwierigkeiten hin, „vergangene Sehgewohnheiten, Sichtweisen und die Evolution innerer Bilder zu rekonstruieren“13.

Mittels Bildanalysen, die verschiedene Medien und Bildkorpora vergleichen, können jedoch Hypothesen aufgestellt werden, welche Nachrichten den unterschiedlichen Rezipienten vermittelt werden sollten. Wiederkehrende Motive, Perspektiven oder Bildnarrative lassen in Verbindung mit Kontextinformationen anderer Quellen Rückschlüsse darauf zu, welche Wirkung entsprechende Fotografien gehabt haben könnten. ${ }^{14}$

\subsection{Die Fabrik als Leitmotiv der russischen Industrialisierung}

Die russische Wirtschaft war im Vergleich zu Westeuropa erst relativ spät auf den Zug der Industrialisierung aufgesprungen. Große gesellschaftliche Veränderungen vollzogen sich im Russischen Reich somit erst im letzten Drittel des 19. und zu Beginn des 20. Jahrhunderts und damit zur selben Zeit, in der sich die Fotografie dort etablierte. Der parallele Verlauf beider Entwicklungen unterscheidet die Situation im Zarenreich von anderen westeuropäischen Staaten mit einer zu dieser Zeit bereits stark entwickelten Industrie. Das russische Beispiel eignet sich daher besonders, um die Wechselwirkungen zwischen Fotografie und Fabriken genauer unter die historiografische Lupe zu nehmen.

Im Fokus der Arbeit steht die Fabrik als Symbol für ein neues, kapitalistisches Wirtschaftssystem. ${ }^{15}$ Gleichzeitig waren Fabriken Sinnbild einer veränderten Lebens- und Arbeitsweise, die im Kontrast zum ländlichen Milieu stand, das das Zarenreich prägte und aus dem die meisten Arbeiter stammten. ${ }^{16}$ In

Wissen. Mittelalterforschung, historische Kulturwissenschaft, Geschichte und Theorie der historischen Erkenntnis, Göttingen 2011, S. 786-807, S. 794-801.

13 Monica Rüthers: Moskau bauen von Lenin bis Chruščev. Öffentliche Räume zwischen Utopie, Terror und Alltag, Wien, Köln, Weimar 2007, S. 57.

14 Vor den einzelnen Kapiteln wird jeweils genauer auf die im Folgenden angewandte Methode und deren Erkenntnisgewinn eingegangen.

15 Gillian Darlay: Factory, London 2003, S. 157; Rudolf Boch: Fabriken, in: Pim den Boer u. a. (Hrsg.): Europäische Erinnerungsorte, Bd. 2. Das Haus Europa, München 2012, S. 535-542, S. 536 .

16 Carsten Goehrke: Russischer Alltag. Eine Geschichte in neun Zeitbildern, Bd. 2. Auf dem Weg in die Moderne, Zürich 2003, S. 324-332. Im Zarenreich war die Arbeiterschaft bis zur Oktoberrevolution sehr klein, Schätzungen zufolge waren 1917 noch zwischen 80 und 9o Prozent der Bevölkerung Bauern. Viele Fabrikarbeiter zogen zwar in die industriellen Zentren, hielten jedoch gleichzeitig eine enge Verbindung zu ihrem dörflichen Herkunftsort aufrecht, so dass sie sowohl urbane wie rurale Elemente und Lebensweisen vereinten. 
den Werkshallen machten Menschen geballt Erfahrungen, ${ }^{17}$ die typisch für die Industrialisierung waren: Sie erlebten in konzentrierter Form eine Beschleunigung von Arbeitsabläufen, sie erfuhren am eigenen Körper, was die Anpassung des Menschen an eine Maschine bedeutete und sie waren mit einer bislang unbekannten Lärmbelastung konfrontiert. ${ }^{18}$

Fabrikfotografien sind auf mehreren Ebenen besonders geeignet, um wirtschaftliche und gesellschaftliche Veränderungen zu untersuchen. Zum einen entstanden die Bilder mithilfe einer neuen technischen Apparatur, der Kamera. ${ }^{19}$ Ihre Produkte, die fotografischen Aufnahmen, waren damals die fortschrittlichsten statischen Abbildungen. Zum anderen zeigen die Fabrikfotografien den Inbegriff des wirtschaftlichen Fortschritts: Maschinen, Produktionsanlagen, rauchende Schornsteine und industriell gefertigte Produkte. In der zweiten Hälfte des 19. Jahrhunderts existierten im Zarenreich sehr unterschiedliche Vorstellungen darüber, welchen politischen und

David Moon: Peasants and Agriculture, in: Dominic Lieven (Hrsg.): The Cambridge History of Russia, Bd. 2. Imperial Russia, 1689-1917, Cambridge 2006, S. 369-393, S. 374. Zu dieser Thematik auch: Reginald E. Zelnik: The Peasant and the Factory, in: Wayne S. Vucinich (Hrsg.): The Peasant in Nineteenth-Century Russia, Stanford 1968, S. 158-190; David Moon: The Russian Peasantry, 1600-1930. The World the Peasants Made, London, New York 1999; Robert E. Johnson: Peasant and Proletarian. The Working Class of Moscow in the Late Nineteenth Century, New Brunswick 1979; Barbara A. Engel: Between the Fields and the City. Women, Work, and Family in Russia, 1861-1914, Cambridge 1994.

17 Der Begriff der Erfahrung beinhaltet das Durchleben und Deuten einer Situation oder Realität. Ute Daniel: Der Krieg der Frauen, 1914-1918. Zur Innenansicht des Ersten Weltkriegs in Deutschland, in: Gerhard Hirschfeld; Gerd Krumeich; Irina Renz (Hrsg.): „Keiner fühlt sich mehr als Mensch ...". Erlebnis und Wirkung des Ersten Weltkriegs, Essen 1997, S. 131-149, S. 131-132. Eine Fotografie stellt die visuelle Reduktion einer mehrdimensionalen Realität auf eine zweidimensionale Abbildung dar. Weil das Betrachten einer Fotografie somit nicht mit dem Durchleben der abgebildeten Situation gleichgesetzt werden kann, wird mit Blick auf die Wirkung von Fotografien der Begriff Erfahrung in dieser Arbeit nicht verwendet. Zur Definition von Erfahrung auch: Nikolaus Buschmann; Horst Carl: Zugänge zur Erfahrung des Krieges. Forschung, Theorie, Fragestellung, in: dies. (Hrsg.): Die Erfahrung des Krieges. Erfahrungsgeschichtliche Perspektiven von der Französischen Revolution bis zum Zweiten Weltkrieg, Paderborn u. a. 2001, S. 11-26, S. $12-21$.

18 Semën Ivanovič Kanatchikov: From the Story of My Life, in: Victoria Bonnell (Hrsg.): The Russian Worker. Life and Labor under the Tsarist Regime, Berkeley u. a. 1983, S. 36-71, S. 50; Lars Bluma; Karsten Uhl (Hrsg.): Kontrollierte Arbeit - disziplinierte Körper? Zur Sozial- und Kulturgeschichte der Industriearbeit im 19. und 20. Jahrhundert, Bielefeld 2012; Thomas Le Roux: Hygienists, Workers' Bodies and Machines in Nineteenth-Century France, in: Lars Bluma (Hrsg.): A History of the Workplace. Environment and Health at Stake, London 2015, S. 85-100.

19 Fotografie und Apparatur. Bildkultur und Fototechnik im 19. Jahrhundert (Heft der Zeitschrift Fotogeschichte. Beiträge zur Geschichte und Ästhetik der Fotografie Jg. 18/1998, Heft 68/69). 
ökonomischen Weg die russische Gesellschaft in die Zukunft wählen sollte. Gerade vor diesem Hintergrund ist es interessant zu untersuchen, welche Spannungsfelder zwischen Lichtbildnern, ${ }^{20}$ Unternehmern und gesellschaftlicher Öffentlichkeit ab den 186oer Jahren entstanden und welche Interessen einzelne Akteure verfolgten. Um ein möglichst differenziertes Bild der Beziehungen zwischen Fotografie und Industrie zu bekommen, beleuchtet die vorliegende Studie fotografische Aufnahmen in verschiedenen Medien.

Bei der Untersuchung von Fotografien aus Unternehmen und von Fabriken drängt sich der Begriff der Industriefotografie auf. Die Bezeichnung ist jedoch mit Vorsicht zu gebrauchen, weil Industriefotografie als Genre im 19. Jahrhundert noch nicht existierte. Es handelt sich bei einer solchen Klassifizierung um eine Rückprojektion. ${ }^{21}$ Auch heute ist der Begriff nicht klar definiert, so dass die vorliegende Arbeit unter Industriefotografie eine breite Palette an Motiven und Bildern fasst, die teilweise auch anderen Genres zugerechnet werden können wie der Landschafts- oder Portraitfotografie. Sie orientiert sich an den Definitionen von Reinhardt Matz und Clemens Zimmermann, erweitert jedoch deren Verständnis von Industriefotografie. Matz bezeichnet alle fotografischen Aufnahmen, die in einem Firmenarchiv gefunden werden können, als Industriefotografien. ${ }^{22}$ Zimmermann erweitert das Spektrum um Fotografien, mit denen Lichtbildner die Auswirkungen der Industrie im urbanen oder ruralen Raum zeigen..$^{23}$ Beide Autoren stellen die Fabrik und den Fabrikanten ins Zentrum ihrer Definition. Die vorliegende Arbeit fasst auch Fotografien Dritter unter den Begriff Industriefotografie, wenn Aufnahmen einer Fabrik gezeigt werden, beispielsweise gedruckte Fotografien auf Ansichtskarten. Die Zusammenstellung des Quellenkorpus geht nicht von einer festen Definition des Genres Industriefotografie oder einer genau festgelegten Bedeutung des Begriffs aus. Ausschlaggebend ist in erster Linie das abgebildete Motiv der Fabrik. Darum erscheint in diesem Fall ein möglichst breites Verständnis von Industriefotografie zielführend. Diesem Begriff zur Seite gestellt

20 Die Begriffe Lichtbildner und Fotograf werden in dieser Arbeit synonym verwendet.

21 Die Verwendungskontexte der Bilder legen nahe, dass es sich um eine Form von Gebrauchsfotografie handelte. Siehe hierzu: Kapitel „Eine Beziehung entwickelt sich Lichtbilder und neue Produktionsweisen“, S. 81-101.

22 Matz: Industriefotografie, S. 9; Reinhard Matz: Industrie in schöner Landschaft mit freundlichen Menschen. Zur historischen Industriefotografie in Tirol, in: Günther Moschig; Gabriele Rath; Susanne Witz Gall (Hrsg.): Industrielle Bildwelten. Tiroler Industrie in zeitgenössischer Fotografie, Innsbruck 2007, S. 23-33, S. 23.

23 Clemens Zimmermann: Zur Definition der Industriefotografie. Von der Hochindustrialisierung bis zu den dreißiger Jahren, in: Hans-Walter Herrmann; Rauner Hudermann; Eva Keller (Hrsg.): Forschungsaufgabe Industriekultur. Die Saarregion im Vergleich, Saarbrücken 2004, S. 375-389, S. 378-379. 
wird die Bezeichnung Fabrikfotografie, die mit synonymer Bedeutung verwendet wird.

Historiker diskutieren immer wieder, wodurch sich Kleinstfabriken von Handwerksbetrieben unterscheiden. ${ }^{24}$ Dies ist besonders im russischen Kontext wichtig, denn im Zarenreich gab es neben wenigen Groß- viele Kleinunternehmen, bei denen die Grenze zum Handwerk fließend war. ${ }^{25}$ In Quellentexten und Namensgebungen von Unternehmen tauchen nebeneinander die Begriffe zavod (Werk/Betrieb), manufaktura (Manufaktur) und fabrika (Fabrik) auf, ohne eindeutig auf einen Industriezweig, ein Produktionsverfahren oder die Größe eines Unternehmens zu verweisen. ${ }^{26}$ Dem Wunsch nach einer klaren Definition folgend, führte die Regierung des Zarenreichs um die Wende zum 20. Jahrhundert formale Kriterien ein, ab wann ein Betrieb als Fabrik zu gelten habe: Demnach musste ein Unternehmen mindestens 16 Personen beschäftigen. ${ }^{27}$ Dieses Kriterium ist jedoch für aktuelle wissenschaftliche Untersuchungen problematisch, da es allein auf die Größe der Belegschaft abzielt

24 Mehrfach wird in der Forschung darauf hingewiesen, dass diese Trennung nicht nur im russischen Kontext problematisch ist. Bernd Bonwetsch: Die russische Revolution 1917. Eine Sozialgeschichte von der Bauernbefreiung 1861 bis zum Oktoberumsturz, Darmstadt 1991, S. 58; Klaus Gestwa: Proto-Industrialisierung in Rußland. Wirtschaft, Herrschaft und Kultur in Ivanovo und Pavlovo, 1741-1932, Göttingen 1999, S. 26. In Deutschland gab es im 19. Jahrhundert ebenfalls noch keine klare Definition, welche Kriterien eine Fabrik ausmachten. Dietrich Hilger: Fabrik, Fabrikant, in: Otto Brunner; Werner Conze; Reinhart Koselleck (Hrsg.): Geschichtliche Grundbegriffe. Historisches Lexikon zur politisch-sozialen Sprache in Deutschland, Bd. 2, Stuttgart ${ }^{2}$ 1979, S. 229-252, S. 246.

25 Insbesondere sowjetische Historiker waren darum bemüht, die Wirtschaft des Zarenreichs als industrialisiert und kapitalistisch darzustellen. Diesen Beweis sollte eine möglichst hohe Anzahl von Fabriken liefern. Mittelgroße Betriebe waren im Russischen Reich eher selten. Bonwetsch: Die russische Revolution 1917, S. 64.

26 Einer groben Tendenz nach bezeichneten fabrika und manufaktura in erster Linie Unternehmen aus der Textilindustrie, zavod trugen häufiger Berg- oder Hüttenwerke sowie metallverarbeitende Betriebe im Namen. Klaus Heller: Geschichte des modernen Unternehmertums in Russland bis 1917, in: Digitales Handbuch zur Geschichte und Kultur Russlands und Osteuropas, Stand: November 2005, S. 10, URL: https://epub.ub.uni-muenchen. de/76o/1/Heller_Unternehmertum.pdf (zuletzt eingesehen am 14.06.16); Roger Portal: Die russische Industrie am Vorabend der Bauernbefreiung, in: Dietrich Geyer (Hrsg.): Wirtschaft und Gesellschaft im vorrevolutionären Rußland, Köln 1975, S. 133-163, S. 140-141.

27 V. Pokrovskij: Fabrika, in: F. A. Brokgaus; I. A. Efron (Hrsg.): Ėnciklopedičeskij slovar', Tom XXXV. Usinskij pograničnyj okrug - Fenol, Sankt-Peterburg 1902, S. 150-168, S. 150; Elise Kimerling Wirtschafter: Social Identity in Imperial Russia, DeKalb 1997, S. 142. In der sowjetischen Forschung war eine ähnliche Definition üblich, nach der ein Betrieb mit 15 Lohnarbeitern eine Manufaktur war. Gestwa: Proto-Industrialisierung, S. 26. Gerade in der sowjetischen Geschichtswissenschaft war es aufgrund der durch Karl Marx geprägten materialistischen Geschichtsauffassung wichtig, das Zarenreich als möglichst stark industrialisiert zu zeigen. 
und nichts über die historischen Gegebenheiten der Produktionsprozesse aussagt. Andere Definitionsmerkmale wie der Grad der Mechanisierung oder die arbeitsteilige Organisation des Herstellungsprozesses fanden keine Beachtung. ${ }^{28}$ Bei einer Untersuchung von Fabriken als Orte, an denen wirtschaftlicher und gesellschaftlicher Wandel in besonderer Form erfahrbar war, macht es jedoch einen entscheidenden Unterschied, wie stark Maschinen in der Produktion zum Einsatz kamen.

Aus diesem Grund war für die Quellenauswahl wichtig, dass in der Produktion der Betriebe zumindest teilweise Maschinen Verwendung fanden. Folglich spielen Handwerk und Kleinbetriebe im Untersuchungskorpus der vorliegenden Arbeit nur eine untergeordnete Rolle. Die Analyse konzentriert sich auf Betriebe der verarbeitenden Industrie, klammert also Fotografien von der Förderung und Gewinnung von Rohstoffen wie Kohle, Roheisen oder Erdöl aus. ${ }^{29}$ Ausschlaggebend für diese Eingrenzung ist, dass Lichtbildner in Bergwerken unter erschwerten Bedingungen fotografieren mussten. ${ }^{30}$ Außerdem war die Arbeit unter Tage noch in weiten Teilen von Handarbeit dominiert. Die Aufnahmen von Ölfeldern und Raffinerien stammten aus der muslimisch geprägten und daher für die Betrachter im europäischen Russland fremdartig anmutenden Peripherie des Imperiums. Sie gingen mit ganz anderen Themen einher, als dies bei Fabriken der Fall war: Viele Lichtbildner und Reisende begeisterte neben der Technik mindestens ebenso sehr die Exotik der Szenerie und der einheimischen Bevölkerung. ${ }^{31}$

28 Stefan Gorißen: Fabrik, in: Friedrich Jaeger (Hrsg.): Enzyklopädie der Neuzeit, Bd. 3, Stuttgart, Weimar 2006, S. 740-747, S. 740. In Westeuropa galt der Einsatz von Maschinen in der Produktion vielfach als Erkennungsmerkmal einer Fabrik. Martina Heßler: Kulturgeschichte der Technik, Frankfurt a. M., New York 2012, S. 43.

29 Bei Unternehmen, die mehrere Standbeine hatten, beschränkt sich die Untersuchung auf die Geschäftszweige der verarbeitenden Industrie.

30 Assegond: La photographie du travail, S. 121, 132-134, 143-145, 156-157; Photographieren im Bergwerk um 1900. Bergwerksphotographie im Oberharz 1890-1910, Katalog zur Ausstellung Photographieren im Bergwerk um 1900 - Bergwerksphotographie im Oberharz 1890-1910 vom 6. November 1998 bis 7. April 1999, Clausthal-Zellerfeld 1998; Francis Pugh: Industrial Image 1843 to 1918, in: Sue Davies; Caroline Collier (Hrsg.): Industrial Image. British Industrial Photography 1843 to 1986, London 1986, S. 8-23, S. 20-21.

31 Damit einhergehende Fragen nach Exotisierung oder Kolonialisierung werden in dieser Arbeit nicht eingehend behandelt. Beispiele für entsprechende Fotografien finden sich beispielsweise in den Beständen des Branobel Digital Archives (BDA). BDA: Nicolas Oleinikoff Collection: o. A.: Photo Albums with the Baku Motifs, o. O. 1890-1893. Häufig erschienen die Bilder von Ölquellen in Zusammenhang von Landschafts- und Reisefotografien, und standen damit in einem anderen Kontext als Aufnahmen russischer Fabriken. BDA: Nicolas Oleinikoff Collection: o. A.: Photo Album (Green) with the Motifs 
Weniger strikt wird das Kriterium der Mechanisierung in den Fallstudien zu Illustrierten und Postkarten angewandt. Selbst- und Fremdzuschreibungen sind dort maßgeblich für die Auswahl der Bilder, also die Verwendung der Begriffe zavod, manufaktura und fabrika in Kombination mit Fotografien von Fabrikmotiven. Die große Anzahl an Aufnahmen machte es unmöglich, jede Firma auf ihren Mechanisierungsgrad hin zu prüfen. Aus diesem Grund umfassen die Studien zu diesen Medien möglicherweise auch Betriebe, in denen die Handarbeit in der Produktion überwog.

Der großen Bandbreite an unterschiedlichen Bildmedien begegnet die Arbeit mit einer Methodenvielfalt, die den einzelnen medienspezifischen Eigenheiten Rechnung trägt. ${ }^{32} \mathrm{Zu}$ Beginn jedes Quellenkapitels findet sich eine Darstellung der im Folgenden angewandten Methoden und Herangehensweisen. In dieser Arbeit wird versucht, wann immer möglich mit Originaldokumenten zu arbeiten. Besonders bei Fotografien macht es einen entscheidenden Unterschied, ob ein Bild als Digitalisat auf einem Computerbildschirm oder als originaler Abzug analysiert wird. ${ }^{33}$ Neben Format, Besonderheiten der technischen Ausführung bei der Bildproduktion oder Gebrauchsspuren reißt eine elektronische Kopie die Bilder aus ihrem Passepartout und ihrem medialen Kontext. Dieser Hintergrund kann jedoch entscheidende Informationen für eine Interpretation liefern. ${ }^{34}$

\section{Archivalische (Nicht-) Überlieferung russischer Industriefotografien}

Auf die Frage nach Industriefotografien aus dem vorrevolutionären Russland reagierte der Archivar im bibliographischen Katalog des Russischen Staatlichen Historischen Archivs in St. Petersburg mit Kopfschütteln: „Sie suchen zu früh. Solche Bilder gibt es erst nach der Oktoberrevolution“, klärte er die ausländische Doktorandin auf, die ihn gerade nach Fabrikfotografien gefragt

from Azerbaijan, Georgia, Armenia, Uzbekistan, Chechenia and Southern Russia, o. O. o. J., S. 28-29.

32 William Mitchell betonte bereits die Verschiedenartigkeit von Bildern, der nur mit einer Methodenvielfalt begegnet werden könne. Siehe hierzu: Martin Schulz: Ordnungen der Bilder. Eine Einführung in die Bildwissenschaft, München ${ }^{2}$ 2009, S. 127. Matz betont, wie wichtig dies auch für Fotografien von Industrieanlagen sei. Matz: Industriefotografie, S. 24. Hierzu auch: Elizabeth Edwards; Janice Hart: Introduction. Photographs as Objects, in: dies. (Hrsg.): Photographs Objects Histories. On the Materiality of Images, New York 2004, S. $1-15$, S. 3 .

34 Zu dieser Problematik auch: Helmut Lethen: Der Schatten des Fotografen. Bilder und ihre Wirklichkeit, Berlin 2014, S. 171-177. 
hatte. ${ }^{35}$ Er war nicht der Einzige, der diese Ansicht vertrat, immer wieder äußerten Gesprächspartner ähnliche Einschätzungen, wenn die Sprache auf das Thema dieser Arbeit kam. Nach wie vor stammen russische fotografische Bildikonen von Arbeitern und Industrieanlagen aus sowjetischer Zeit. Sie dominieren den russischen Bildkanon und lassen kaum Raum für Fabrikfotografie aus der Zarenzeit.

Mit verantwortlich für die geringe Beachtung der frühen russischen Fabrikfotografie war das mangelnde Interesse der Propagandaschaffenden in der Sowjetunion. In den 196oer bis 1980er Jahren lag ihnen wenig daran zu zeigen, dass es bereits im Zarenreich große Industriebetriebe gegeben hatte. ${ }^{36}$ Ein weiterer Grund liegt darin, dass viele Akten nicht über gängige Bibliothekskataloge gefunden werden können, sondern spezielle Suchanfragen gestellt werden müssen. ${ }^{37}$ Für eine Studie zu russischen Industriefotografien reicht es daher nicht aus, das Zentrale Staatliche Archiv für Kino und Fotodokumente in Moskau und das Zentrale Staatliche Archiv für Kino-, Fono- und Fotodokumente in St. Petersburg zu konsultieren und sich auf Findbücher zu verlassen. Stattdessen ist es eine zentrale Aufgabe des Historikers zu überlegen, in welchen Akten und in Zusammenhang mit welchen Themen und möglicherweise überlieferten Dokumenten Fotografien eine Rolle gespielt haben könnten, um Aufnahmen und Kontextinformationen ausfindig zu machen. ${ }^{38}$

35 Der Besuch fand am 3. September 2012 statt. Später stellte sich heraus, dass es sogar in den Beständen des RGIA Fabrikfotografien gibt. Beispielsweise: RGIA f. 37, op. 81, d. 224: o. A.: Vidy chimičeskich zavodov Tovariščestva P. K. Uškova i Ko. Bliz goroda Eladugi Vjatekoj gub., na reke Kam, Kazan' o. J.

36 In Firmenfestschriften aus dieser Zeit fehlen häufig vorrevolutionäre Fabrikfotografien vollständig. Semen Abramovič Gol'denberg; Jakov Moiseevič Gammerman: Bal'tijskaja manufaktura. 1898-1968, Tallin 1969; T. M. Gudkovkaja u. a. (Hrsg.): Konditerskoj fabrike „Krasnyj Oktjabr'“ 100 let. 1867-1967, Moskva 1967; Viktor Andreevič Babičev: V sojuze s ènergetikoj. K 125-letiju zavoda, Jaroslavl' 1986. Teilweise wird nur eine Hütte aus rohen Brettern gezeigt, in der im Zarenreich angeblich die Arbeiter gewohnt hätten. Die Fotografien solcher Hütten gleichen sich in unterschiedlichen Veröffentlichungen erstaunlich stark, was nahelegt, dass es sich um Aufnahmen handelt, die erst in der Sowjetunion zu Propagandazwecken angefertigt worden waren. Entsprechende Fotografien zeigen: T. P. Bulavina; Z. D. Makarova: Slavnaja istorija. Očerki istorii Vyksun. ordena Lenina metallurg. zavoda. 1757-1967, Gor'kij 1967, S. 99; L. I. Novinkov: Zavod i Puti. K 100-letiju Volgogr. derevoobdeločnogo zavoda im. V. V. Kujbyševa, Volgograd 1970, S. 19; o. A.: Ižorskij zavod: K 25o-letiju so dnja osnovanija zavoda, Leningrad 1972, S. 46.

37 Dies war in den RNB, RGB, NBGÉ ORK der Fall.

38 Zur Thematik russischer Archivpraktiken siehe auch: Julia Herzberg: Gegenarchive. Bäuerliche Autobiographik zwischen Zarenreich und Sowjetunion, Bielefeld 2013, S. $65-83$. 
Bestimmte Lücken im Quellenkorpus lassen sich dennoch nicht vermeiden. Kleinstbetriebe beispielsweise hinterließen oft nur wenige Dokumente und fast nie fotografische Zeugnisse. Leider zählen hierzu auch Fotoateliers. Von keinem Studio aus dem Zarenreich konnten Dokumente wie Rechenbücher oder Abbildungsverzeichnisse ausfindig gemacht werden, aus denen die alltäglichen Abläufe rekonstruiert werden könnten. In der Sekundärliteratur fehlen entsprechende Hinweise ebenfalls.

Im Quellenkorpus der Arbeit dominieren daher große Unternehmen mit mehreren hundert Beschäftigten. Für kleine Unternehmer waren aufwendige Bilddokumentationen nicht erschwinglich. Hinzu kommt, dass Firmenarchive der beste Garant für das Überleben von Bildern waren. Kleine Betriebe verfügten jedoch kaum über solche Einrichtungen. Vorzeigebeispiel eines Firmenarchivs mit beeindruckenden fotografischen Beständen ist in Deutschland das Historische Archiv Krupp. Gegründet Anfang des 20. Jahrhunderts, ${ }^{39}$ umfassen die Bestände hunderte Aufnahmen aus der zweiten Hälfte des 19. Jahrhunderts. Die Friedrich Krupp AG begann schon früh mit der Archivierung ihrer fotografischen Abzüge und Negative, so dass neben repräsentativen Alben auch einzelne Fotografien aus der Produktion, von Baumaßnahmen oder Fabrikbesuchen überliefert sind. Die meisten Betriebe, auch außerhalb des Zarenreichs, verfügten Anfang des 20. Jahrhunderts nicht über einen solch großen Bestand an Aufnahmen, darüber hinaus ließen sie es an einer geordneten Archivierung fehlen. ${ }^{40}$ Insbesondere lose Einzelaufnahmen verloren, einmal aus ihrem Entstehungszusammenhang gerissen, leicht ihren Kontext und damit ihre Bedeutung. Es ist davon auszugehen, dass die geringe Anzahl an Einzelaufnahmen in Archiven russischer Firmen nicht daraus resultiert, dass es diese Bilder nicht gab, sondern dass sie verloren gingen oder zerstört wurden.

Nachdem die Bolschewiki 1917 die Macht an sich gerissen hatten, verstaatlichten sie die ehemals privaten Unternehmen im Zarenreich. Ein Teil von deren Dokumenten fand ihren Weg in staatliche Archive. ${ }^{41}$ Dieser Übergang barg Gefahren: Nicht alle Archivare beließen Fotografien bei den zugehörigen Dokumenten. Manche kassierten die Aufnahmen, weil sie ihnen

39 Klaus Tenfelde: Im Zenit industriepolitischer Macht. Die Jahrhundertfeier der Fried. Krupp AG 1912, in: Paul Münch (Hrsg.): Jubiläum, Jubiläum ... Zur Geschichte öffentlicher und privater Erinnerung, Essen 2005, S. 109-143, S. 137-138.

40 Siehe hierzu: Kapitel „Neue Bilder eines neuen Raums - Fabrikalben“, S. 103-178.

41 Ein weiterer Fundort für Firmenfotografien aus dem Zarenreich sind die Archive derjenigen Unternehmen, die russische Niederlassungen unterhielten. Die Dokumente der russischen Dependenzen befinden sich zwar auch in russischen Archiven. Aufnahmen, die vor der Oktoberrevolution an das Mutterunternehmen geschickt wurden, befinden sich heute jedoch in Archiven in Westeuropa. 
wertlos erschienen. ${ }^{42}$ Archive konnten Bilder einerseits bewahren, sie konnten andererseits je nach Archivierungsvorgaben Fotografien aber auch atomisieren und aus ihrem Kontext reißen. Susan Sontag geht auf diese Problematik ein, wenn sie schreibt: „Genau genommen lässt sich aus einem Foto nie etwas verstehen. ${ }^{43}$ Ohne Hintergrundinformationen verlieren fotografische Aufnahmen wichtige Aspekte ihrer Bedeutung sowie ihres Interpretations- und Vermarktungspotentials, die sie erhaltenswürdig machen und schützen. ${ }^{44}$ Hinzu kommen gerade im Untersuchungsgebiet der vorliegenden Arbeit militärische Auseinandersetzungen wie der Erste Weltkrieg, der russische Bürgerkrieg sowie der Zweite Weltkrieg, die eine große Bedrohung für Bildbestände und Firmennachlässe waren. Während die sowjetische Regierung die Produktionsanlagen im Zweiten Weltkrieg nach Osten evakuierte, war die Sicherung der Firmenarchive eher nachrangig. Ihnen konnten Kampfhandlungen zum Verhängnis werden, andere Akten gingen bei der Evakuierung von Archivbeständen unwiederbringlich verloren.

Fabrikfotografien werden auch in Bibliotheken, Privatsammlungen und Firmenmuseen aufbewahrt. Als in der Nachkriegszeit sowjetische Großbetriebe eigene Museen gründeten, wandten sie sich mit Aufrufen an ehemalige Mitarbeiter und baten diese um Bilder und Dokumente aus der Geschichte des Unternehmens. ${ }^{45}$ Solche Initiativen waren nur Betrieben möglich, die auch nach dem Zweiten Weltkrieg über genügend Kapital verfügten. Hieraus erklärt

42 Für diese Annahme spricht der Vergleich zwischen den Stadtarchiven in Moskau (Moskvy Central'nyj Gosudarstvennyj Archiv - CGA) und St. Petersburg (Central'nyj Gosudarstvennyj Istoričeskij Archiv Sankt Peterburga - CGIA). In beiden Archiven finden sich Firmennachlässe. Doch während in Moskau Fotografien auch Teil von Dossiers mit alltäglicher Firmenkorrespondenz sind, umfassen die Bestände in St. Petersburg nur vereinzelt Fotoalben und keine einzelnen Abzüge von Unternehmen. Dieses Quellenproblem lässt sich auch außerhalb Russlands beobachten. Ulrich Pohlmann; Rudolf Scheutle: Vorwort, in: dies. (Hrsg.): Industriezeit. Fotografien 1845-2010, Tübingen 2011, S. 6.

43 Susan Sontag: In Platos Höhle (1977), in: Bernd Stiegler (Hrsg.): Texte zur Theorie der Fotografie, Stuttgart 2010, S. 277-301, S. 300.

44 Zur Rolle, die Archive mit Blick auf Fotografien einnehmen: Allan Sekula: Reading an Archive. Photography between Labour and Capital, in: Jessica Evans; Stuart Hall (Hrsg.): Visual Culture. The Reader, London ${ }^{2} 2001$, S. 181-192, S. 184-185.

45 Das Muzej istorii OAO Ižorskie zavody sowie das Muzej Kirovskogo zavoda wandten sich an ehemalige Mitarbeiter mit der Bitte, ihnen Dokumente aus der Firmengeschichte zur Verfügung zu stellen. Auch in der DDR waren Fabrikfotografien und Dokumente eines Unternehmens häufig nicht im gleichen Archiv, sondern verstreut über mehrere Einrichtungen. Petra Clemens: „Betriebsgeschehen“ im VEB - in Fotos und beim Fotografieren, in: Irene Ziehe, Ulrich Hägele (Hrsg.): Fotografien vom Alltag - Fotografieren als Alltag, Münster 2004, S. 249-260, S. 250. 
sich, weshalb die Metall- und Schwerindustrie im vorliegenden Quellenkorpus so stark vertreten ist: Im Zarenreich zählten viele Firmen der metallverarbeitenden Industrie bereits zu den Großunternehmen, deren Anlagen das Interesse der Fotografen weckten und deren Inhaber offen für neue Medien waren. Nach der Oktoberrevolution, insbesondere mit Beginn der forcierten Industrialisierung Stalins ab 1929, spielte die Schwerindustrie eine wichtige Rolle in der Propaganda der Bolschewiki. Die neue politische Führung wies ihr bei der Inszenierung von Infrastruktur- und Modernisierungsprojekten eine Hauptrolle zu und machte sie zu einer Vorzeigebranche. ${ }^{46}$ Im Gegensatz zu Fabriken der Textil- oder Konsumgüterindustrie überstanden diese Firmen auch das Ende der Sowjetunion vergleichsweise gut, so dass ein großer Teil dieser Unternehmen bis heute besteht und die Bildbestände sichern konnte. ${ }^{47}$

\subsection{Untersuchungszeit und Untersuchungsraum}

Das Aufkommen erster Fabrikfotografien in den 186oer Jahren und die Oktoberrevolution bilden den zeitlichen Rahmen der Arbeit. Zwar kann die Frage aufgeworfen werden, ob sich die Oktoberrevolution als Zäsur für eine kulturwissenschaftliche Untersuchung eignet. Die Bolschewiki setzten die Industrie in ihrer Ideologie jedoch so zentral, dass sich das ideologische Passepartout der Fabrikfotografien nach dem Machtwechsel völlig änderte. ${ }^{48}$ Dies rechtfertigt 1917 als Endpunkt der Hauptanalyse. Die Ergebnisse ausgewählter Fallstudien zur Sowjetischen Fabrikfotografie der 1920er und 1930er Jahre werden in einem

46 Klaus Gestwa: Die Stalinschen Großbauten des Kommunismus. Sowjetische Technik- und Umweltgeschichte, 1948-1967, München 2010, S. 251-255.

47 Das Beispiel der Gummifabrik Treugol'nik in St. Petersburg unterstreicht, wie wichtig die Existenz eines Unternehmens für den Fortbestand seiner historischen Dokumente ist. Die im Zarenreich gegründete Fabrik hatte ihren Sitz in St. Petersburg und produzierte bis 2001 Artikel aus Gummi. Nachdem 2005 das Insolvenzverfahren abgeschlossen war, wurde das Firmenmuseum aufgelöst. Seitdem ist unklar, wo sich die Museumsbestände heute befinden. Auch von den Zucker- und Samowarfabriken der Stadt Tula, in denen 1913 teilweise mehrere hundert Menschen arbeiteten, sind sehr wenige Fotografien überliefert. o. A.: Spisok fabrik, zavodov i dr. promyšlenny cipredprijatij Tul'skoj gubernii. Po dannym Vserossijskoj Promyšlennoj i Professional'noj perepisi 1918 goda, Tula 1918, S. 8-9, 20-21.

48 Zur speziellen Rolle, die die Industrie in der Propaganda der Bolschewiki einnahm: Peter Gatrell: Reconceptualizing Russia's Industrial Revolution, in: Jeff Horn, Leonard Rosenband, Merritt Smith (Hrsg.): Reconceptualizing the Industrial Revolution, Cambridge, Mass. 2010, S. 229-249, S. 242-243. 
kurzen Abschlusskapitel präsentiert. Sie legen dar, wie sehr sich die Industriefotografie in der jungen Sowjetunion veränderte.

Geographisch konzentriert sich die Arbeit auf Industriebetriebe im europäischen und russischsprachigen Teil des Zarenreichs. ${ }^{49}$ Die Untersuchung von Bilddiskursen zu wichtigen Industriezentren des Zarenreichs, wie zum Königreich Polen, muss zukünftigen Forschungsarbeiten vorbehalten bleiben. Immer wieder weisen Blickwinkel sowie Themen Aspekte auf, die über die Grenzen des Russischen Reichs hinaus weisen, beispielsweise die Entwicklung der Fabrikfotografie während des Ersten Weltkriegs. Dabei wird herausgearbeitet, in welchen internationalen Kontexten und Netzwerken sich Fotografen und ihre Bilder bewegten.

Im Zarenreich fand die erste Welle der Mechanisierung der Industrie und der damit einhergehende Strukturwandel in der ersten Hälfte des 19. Jahrhunderts in der Baumwoll- und Textilindustrie statt. Die Mehrheit der betroffenen Unternehmen produzierte in kleineren Städten oder Gewerbedörfern. ${ }^{50}$ Im Quellenkorpus der vorliegenden Arbeit sind sie im Vergleich zu Betrieben aus Moskau und St. Petersburg unterrepräsentiert. Zum Zeitpunkt dieser Mechanisierung war die Fotografie noch kein Bildmedium, das Fabrikanten verwendeten. Mehrere Jahrzehnte später, als sich die neue Bildtechnik durchgesetzt hatte, waren diese Betriebe bereits keine Vorzeigeunternehmen mehr. Stattdessen hatten sich gerade in der Hauptstadt des Zarenreichs die Metallverarbeitung, Elektro- und Chemieindustrie zu stark industrialisierten Branchen entwickelt. Mit ihrer fortschrittlichen, technischen Ausstattung erwirtschafteten sie hohe Gewinne und verfügten somit über die Mittel, um Fotografien zu finanzieren. Außerdem waren ihre Besitzer offen für neue Präsentationsformate. ${ }^{51}$ Die vorliegende Arbeit unterzieht die Beziehung zwischen Industrie und Fotografie einer ersten Analyse und nutzt als Grundlage hierfür eine breite Quellenbasis, die sowohl unterschiedliche Standorte als auch Industriezweige berücksichtigt.

49 Grund hierfür sind pragmatische Überlegungen; sehr weit auseinanderliegende Archive hätten die Bearbeitung des Themas in der vorgesehenen Zeit unmöglich gemacht.

50 Bonwetsch: Die russische Revolution 1917, S. 57-61. Ein großer Teil dieser Betriebe lag in den Regionen um Moskau und St. Petersburg. Dietmar Neutatz: Träume und Alpträume. Eine Geschichte Russlands im 20. Jahrhundert, München 2013, S. 79; William Blackwell: The Beginnings of Russian Industrialization 1800-186o, Princeton 1968, S. 42-43.

51 Bonwetsch: Die russische Revolution 1917, S. 66. Die vorliegende Arbeit unterzieht die Beziehung zwischen Industrie und Fotografie einer ersten Analyse, die sich auf eine breite aber nur qualitative Auswahl an Fotografien stützt. Es wäre aufschlussreich in einem weiteren Schritt, die Ergebnisse der Untersuchung anhand von Lokalstudien zu überprüfen, um gegebenenfalls Abweichungen oder Besonderheiten herauszuarbeiten. 


\subsection{Forschungskontext: Geschichte der russischen Industrialisierung}

Die beiden Begriffe Fotografie und Industrie im Titel markieren zwei Forschungsfelder, die die Arbeit stark prägen: Industrie steht für Ökonomie und Wirtschaftsgeschichte, Fotografie für visuelle Geschichte als Teil der neuen Kulturgeschichte. Es handelt sich bei der vorliegenden Arbeit um eine kulturwissenschaftliche Studie, die Forschungen zur Geschichte der Industrialisierung bilden jedoch das Fundament, auf das sich die Untersuchung stützt. ${ }^{52}$ Bis heute sind die Forschungen Alexander Gerschenkrons zur russischen Industrialisierung Bezugspunkt auch neuerer Arbeiten zur Wirtschaft im Zarenreich. ${ }^{53}$ Gerschenkrons These der Rückständigkeit der russischen Ökonomie ${ }^{54}$ rief heftige Reaktionen hervor. ${ }^{55}$ Noch immer wird die Frage nach Rückständigkeit oder Fortschrittlichkeit des vorrevolutionären Russlands häufig gestellt. ${ }^{56}$ Allerdings interessieren sich die Autoren weniger für genaue Abwägungen, ob das Russische Reich gegenüber Westeuropa

$5^{2}$ Die Hochzeit der Forschungen zur Wirtschaftsgeschichte Russlands waren die 196oer bis 1980er Jahre.

53 Dies skizziert: Manfred Hildermeier: Geschichte Russlands. Vom Mittelalter bis zur Oktoberrevolution, München 2013, S. 1130-1131, 1139, 1154-1156.

54 Alexander Gerschenkron: Economic Backwardness in Historical Perspective. A Book in Essays, Cambridge, Mass. 1962, besonders der Titelaufsatz S. 5-30, S. 6-11, 16-22.

55 Einen Überblick über die Entwicklung des Begriffs der Rückständigkeit gibt Manfred Hildermeier: Das Privileg der Rückständigkeit. Anmerkungen zum Wandel einer Interpretationsfigur der neueren russischen Geschichte, in: Historische Zeitschrift, Jg. 244/1987, Heft 3, S. 557-603. Aktuelle Auseinandersetzungen finden sich in: David Feest; Lutz Häfner (Hrsg.): Die Zukunft der Rückständigkeit. Chancen - Formen - Mehrwert. Festschrift für Manfred Hildermeier zum 65. Geburtstag, Köln, Weimar, Wien 2016; Hildermeier: Geschichte Russlands, besonders S. 1317-1346. William Blackwell widerlegte Gerschenkrons These, der zufolge es vor der Bauernbefreiung in Russland keinerlei Industrie gegeben habe. Blackwell: The Beginnings of Russian Industrialization, S. 37. Weitere Studien betonten, dass neben den Gründungen von Großunternehmen die Bedeutung von Kleinbetrieben und Heimarbeit nicht unterschätzt werden dürfe. Das Kustargewerbe trug erheblich zu den wirtschaftlichen Einnahmen des Zarenreichs bei und behauptete sich im langen 19. Jahrhundert auch neben großen Manufakturen und Fabriken. Gatrell: Reconceptualizing Russia's Industrial Revolution, S. 229. Speziell zum Kustargewerbe: Gestwa, Proto-Industrialisierung. Zu Beginn des 20. Jahrhundert machte es noch etwa ein Drittel der gesamten Industrieproduktion im Zarenreich aus. Raymon W. Goldsmith: The Economic Growth of Tsarist Russia 1860-1913, in: Economic Development and Cultural Change, Jg. 9/1961, Heft 3, S 441-475, S. 468.

56 Zu dieser Thematik: Frithjof Benjamin Schenk: Russlands Aufbruch in die Moderne? Konzeptionelle Überlegungen zur Beschreibung historischen Wandels im Zarenreich im 19. Jahrhundert, in: Martin Lengwiler; Christof Dejung (Hrsg.): Ränder der Moderne. Neue Perspektiven auf die Europäische Geschichte (1800-1930), Köln, Weimar, Wien 2016, S. $183^{-203}$, S. $185^{-192 .}$ 
rückständig war oder nicht. Stattdessen untersuchen sie, welchen Weg in die Zukunft und eine wie auch immer geartete Moderne das Zarenreich angesichts massiver gesellschaftlicher Veränderungen wählte. ${ }^{57}$ Sie zeichnen nach, auf welche Weise der Ausbau von Infrastruktur sowie das Aufkommen neuer technischer Errungenschaften wie der Eisenbahn oder der Telegraphie zu neuen Kommunikationsformen und Wahrnehmungsweisen führten. ${ }^{58}$ Die vorliegende Studie erweitert diese Untersuchungen mit der Analyse von Fotografien um den Aspekt visueller Kommunikationstechniken.

Darüber hinaus fügt die Arbeit einen neuen Aspekt zum in der Wirtschaftsgeschichte viel diskutierten Themenkomplex der Bedeutung staatlichen Engagements für die Entwicklung der russischen Industrialisierung hinzu. ${ }^{59}$ Sie zeigt, dass staatliche wie private Industrielle gleichermaßen offen für neue Medien waren und durch finanzielles Engagement teilweise sogar einen Beitrag zur Weiterentwicklung der Fotografie leisteten. Innerhalb dieser Arbeit wird bei der Bildanalyse nur in Einzelfällen zwischen staatlichen und privaten Betrieben unterschieden. Stichproben bei Großbetrieben beider Eigentumsformen ergaben kaum Unterschiede im Umgang mit ihren Fotografien..$^{60}$ Entscheidend war vielmehr die Größe des Unternehmens.

57 In diesem Kontext steht auch das Forschungsprojekt Russlands Aufbruch in die Moderne. Technische Innovation und die Neuordnung sozialer Räume im 19. Jahrhundert an der Universität Basel, im Rahmen dessen die vorliegende Arbeit entstand.

58 Frithjof Benjamin Schenk: Russlands Fahrt in die Moderne. Mobilität und sozialer Raum im Eisenbahnzeitalter, Stuttgart 2014; Walter Sperling: Der Aufbruch der Provinz. Die Eisenbahn und die Neuordnung der Räume im Zarenreich, Frankfurt a. M., New York 2011; Roland Cvetkovski: Modernisierung durch Beschleunigung. Raum und Mobilität im Zarenreich, Frankfurt a. M., New York 2006. Zu Kommunikation in Osteuropa: Walter Sperling (Hrsg.): Jenseits der Zarenmacht. Dimensionen des Politischen im Russischen Reich 1800-1917, Frankfurt a. M., New York 2008; Christophe von Werdt: Kommunikation (oder Einleitung), in: Nada Boškovska u. a. (Hrsg.): Wege der Kommunikation in der Geschichte Osteuropas, Köln, Weimar, Wien 2002, S. IX-XX.

59 Arcadius Kahan: Wirtschafts- und Sozialgeschichte Rußlands und Kongreßpolens 186o1914, in: ders. u. a. (Hrsg.): Handbuch der europäischen Wirtschafts- und Sozialgeschichte: Ost- und Südosteuropa, 1850-1914, Stuttgart 1980, S. 7-78, S. 76. Wayne Dowler betont, dass in westeuropäischen Staaten die Entwicklung der Eisenbahn in völlig vergleichbarem Rahmen erfolgte. Der russische Staat habe sich nur in der Rüstungsindustrie mit einer ungewöhnlich starken Förderung engagiert. Wayne Dowler: Russia in 1913, DeKalb ${ }^{2}$ 2012, S. 45-46. Zur Bedeutung von ausländischem und privatem Kapital auch: John P. McKay: Pioneers for Profit. Foreign Entrepreneurship and Russian Industrialization 1885-1913, Chicago 1970.

6o Teilweise wechselten Fabriken im Untersuchungszeitraum auch mehrfach ihren Status, gingen bankrott, wurden verstaatlicht und wieder an private Unternehmer verkauft. Diese Prozesse machen eine strikte Trennung zwischen den Organisationsformen zusätzlich schwierig. Ein Beispiel hierfür sind die Kirovskogo zavoda. Jonathan A. Grant: Big 
Ende der 1980er Jahre ging das Forschungsinteresse an Wirtschaftsgeschichte langsam zurück. In der osteuropäischen Geschichte erlebte das Feld in den 1990er Jahren jedoch einen neuen Aufschwung, als russische Archive den Zugang für ausländische Wissenschaftler erleichterten und neue Quellenbestände zugänglich machten. Es entstanden Arbeiten, die sich für soziale Identität, das Alltagsleben der Arbeiter oder Einzelpersonen interessierten. ${ }^{61}$ Während in den 20ooer Jahren international das historiografische Interesse am 19. Jahrhundert wieder erwachte, ${ }^{62}$ erschienen in der osteuropäischen Geschichte nur wenige Arbeiten zur Industrialisierung. ${ }^{63}$ Es entstanden Studien zur Ideengeschichte der Industrialisierung beziehungsweise dem zeitgenössischen Blick auf die Diskurse über Industrie. ${ }^{64}$ Was bislang jedoch fehlt, sind Arbeiten, die die russische Industrialisierung aus der Perspektive der neuen Kulturgeschichte, speziell der visuellen Geschichte heraus untersuchen. Die vorliegende Arbeit trägt dazu bei, die Industrialisierungsforschung mit dem Feld der visuellen Geschichte, speziell der Geschichte der Fotografie, zu verbinden. Die Historikerin Monika Dommann fordert bereits seit einigen Jahren, dass sich auch Geisteswissenschaftler Themen zuwenden sollten, die von den Wirtschaftswissenschaften dominiert seien. ${ }^{65}$ Dommann untersucht besonders Fragen zu Kulturtechniken und ruft zur Analyse ökonomischer Praktiken auf. Hierzu zählt auch die Fotografie, beziehungsweise

Business in Russia. The Putilov Company in Late Imperial Russia, 1868-1917, Pittsburgh 1999.

61 Beispielsweise: Mark Steinberg: Moral Communities. The Culture of Class Relations in the Russian Printing Industry, 1867-1907, Berkeley 1992; Gestwa, Proto-Industrialisierung; Engel: Between the Fields and the City; James West; Iurii Petrov: Merchant Moscow. Images of Russia's Vanished Bourgeoisie, Princeton 1998; Dittmar Dahlmann u. a. (Hrsg.): „Eine große Zukunft“. Deutsche in Russlands Wirtschaft, Berlin, Bonn, Gießen u. a. 200o; Rainer Lindner: Unternehmer und Stadt in der Ukraine, 1860-1914. Industrialisierung und soziale Kommunikation im südlichen Zarenreich, Konstanz 2006.

62 Stellvertretend hierzu die Publikationen von: Christopher Bayly: Die Geburt der modernen Welt. Eine Globalgeschichte 1780-1914, Frankfurt a. M., New York 2008; Osterhammel: Verwandlung der Welt.

63 Es erschienen zwar Arbeiten zur Industrialisierung, aber oft waren es Publikationen von Autoren, die sich dem Thema bereits vor dem Zerfall der Sowjetunion widmeten. So: Peter Gatrell: The Tsarist Economy 1850-1917, London 1986; Gatrell: Reconceptualizing Russia's Industrial Revolution.

64 Beispielsweise: Dowler: Russia in 1913, besonders S. 234-245; Wayne Dowler: The Intelligentsia and Capitalism, in: William Leatherbarrow; Derek Offord (Hrsg.): A History of Russian Thought, Cambridge u. a. 2010, S. 263-285; Vincent Barnett: A History of Russian Economic Thought, London, New York 2005.

65 Monika Dommann: Reden wir über Geld! Aber wie? Und wozu?, in: Zeitschrift für Kulturwissenschaften, Jg. 5/2011, Heft 1, S. 113-121, S. 118. 
die Verwendung von Bildern. ${ }^{66}$ Gerade Fabrikfotografien eignen sich daher, das empirische und interpretatorische Potential der kulturwissenschaftlichen Forschung für die Wirtschaftsgeschichte fruchtbar zu machen.

\subsection{Forschungskontext: Visuelle Geschichte des ausgehenden Zarenreichs}

Der Bereich der visuellen Geschichte ist im Gegensatz zur Geschichte der Industrialisierung ein junges Forschungsfeld. Lange verwendeten Historiker Bilder nur, um ihre Arbeiten zu illustrieren. Erst mit der Verbreitung der neuen Kulturgeschichte in den 1990er Jahren änderte sich dies langsam. Die kulturwissenschaftliche Forschung fragt, wie Menschen sich in der Vergangenheit ihre Wirklichkeit über konstruierte Bedeutungskontexte und Sinnmuster aneigneten. ${ }^{67}$ In diesem Zuge fand eine Neubewertung bildlicher Quellen statt. Nach der Ausrufung des iconic oder visual turn ${ }^{68}$ wandten sich in den letzten Jahren Historiker in ihren Arbeiten vermehrt visuellen Quellen zu, ein Trend, der auch in der osteuropäischen Geschichte zu beobachten ist. ${ }^{69}$ Der

66 Monika Dommann; Daniel Speich Chassé; Mischa Suter: Einleitung. Wissensgeschichte ökonomischer Praktiken, in: Berichte zur Wissenschaftsgeschichte, Jg. 37/2014, Heft 2, S. 107-111, S. 109-110.

67 Achim Landwehr: Kulturgeschichte. Version: 1.o, in: Docupedia-Zeitgeschichte, 14.5.2013, URL: http://docupedia.de/zg/Kulturgeschichte (zuletzt eingesehen am 31.02.2017).

68 Doris Bachmann-Medick: Cultural Turns. Neuorientierungen in den Kulturwissenschaften, Reinbek bei Hamburg 2006, S. 329-348, 360-365; Gottfried Boehm: Die Wiederkehr der Bilder, in: ders. (Hrsg.): Was ist ein Bild? München 1994, S. 11-38, S. 13-17; Gottfried Boehm: Iconic Turn. Ein Brief, in: Hans Belting (Hrsg.): Bilderfragen. Die Bildwissenschaften im Aufbruch, München 2007, S. 27-36; Peter Burke: Augenzeugenschaft. Bilder als historische Quellen, Berlin 2010, S. 10-21; Heinz Dieter Kittsteiner: „Iconic Turn“ und „innere Bilder“ in der Kulturgeschichte, in: ders. (Hrsg.): Was sind Kulturwissenschaften? 13 Antworten, München 2004, S. 153-182, S. 162-165; Gerhard Paul: Visual History. Version: 3.0, in: Docupedia-Zeitgeschichte, 13.3.2014, URL: http://docupedia.de/ zg/Visual_History_Version_3.o_Gerhard_Paul (zuletzt eingesehen am 31.03.2017); Gerhard Paul: Von der Historischen Bildkunde zur Visual History, in: ders. (Hrsg.): Visual History. Ein Studienbuch, Göttingen 2006, S. 7-36; William J. T. Mitchell: Bildtheorie, Frankfurt a. M. 2008, S. 106-108; William J. T. Mitchell: Pictorial Turn. Eine Antwort, in: Hans Belting (Hrsg.): Bilderfragen. Die Bildwissenschaften im Aufbruch, München 2007, S. 37-46.

69 Beispielsweise: Isabelle de Keghel; Andreas Renner (Hrsg.): Fotografie in Russland und der Sowjetunion, Themenheft: Fotogeschichte. Beiträge zur Geschichte und Ästhetik der Fotografie, Jg. 35/2015, Heft 136; Valerie Kivelson; Joan Neuberger (Hrsg): Picturing Russia. Explorations in Visual Culture, New Haven 2008; Klaus Gestwa; Katharina Kucher (Hrsg.): Visuelle Geschichte Russlands im 19. Jahrhundert, Themenheft: Jahrbücher für Geschichte Osteuropas, Jg. 6o/2012, Heft 4; Alexander Kraus; Andreas Renner (Hrsg.): 
Schwerpunkt der Untersuchungen zum Zarenreich und der Sowjetunion lag häufig auf Gemälden oder Plakaten, ${ }^{70}$ doch auch Fotografien rückten verstärkt in den Fokus der historischen Bildforschung: Besonderer Beliebtheit erfreuten sich die sowjetische Fotografie der Avantgarde ${ }^{71}$ und Pressefotografien. ${ }^{72}$

Das große Interesse einer breiten Öffentlichkeit für russische Fotografie, insbesondere aus dem vorrevolutionären Russland, schlug sich seit dem Ende der Sowjetunion in der Russischen Föderation in einer Flut von Bildbänden und zahlreichen Ausstellungen nieder. In den 20ooer Jahren begannen viele russische Wissenschaftler, sich mit fotografischen Aufnahmen aus dem Zarenreich auseinanderzusetzen. Es erschienen mehrere Standardwerke zur Geschichte der russischen Fotografie ${ }^{73}$ sowie illustrierte Nachschlagewerke, die Informationen zu Fotografen einer Stadt oder einer Straße zusammentrugen. ${ }^{74}$ Bis heute setzen sich viele Analysen mit den Werken bekannter Fotografen, Entwicklungen in der Kunstfotografie oder mit der fotografischen

Geschichte sehen. Historische Bildforschung und osteuropäische Geschichte, Ausgabe Zeitenblicke, Jg. 10/2011, Nr. 2, URL: http://www.zeitenblicke.de/2011/2/Einfuehrung/ (zuletzt eingesehen am 08.07.16); Igor' Vladimirovič Narskij: Očevidnaja istorija. Problemy vizual'noj istorii Rossii XX stoletija, Čeljabinsk 2008. Für einen Überblick über die Forschungen speziell zur Fotografie: Andreas Renner: Der Visual Turn und die Geschichte der Fotografie im Zarenreich und in der Sowjetunion, in: Jahrbücher für Geschichte Osteuropas, Jg. 62/2014, Heft 3, S. 401-424.

70 Klaus Waschik; Nina Baburina (Hrsg.): Werben für die Utopie. Russische Plakatkunst des 20. Jahrhunderts, Bietigheim-Bissingen 2003; Katharina Kucher: Die visualisierte Kindheit im Russland des 19. Jahrhunderts. Stilisierte Welten zwischen Rückständigkeit und Modernisierung, in: Jahrbücher für Geschichte Osteuropas, Jg. 6o/2012, Heft 4, S. 510-532; Ada Raev: Die Portraitgalerie bedeutender Zeitgenossen von Pavel Tret'jakov als Spiegel des sich wandelnden Selbstverständnisses der russischen Gesellschaft im 19. Jahrhundert, in: Jahrbücher für Geschichte Osteuropas, Jg. 6o/2012, Heft 4, S. 561-589.

71 Beispielsweise: Elliott (Hrsg.): Photography in Russia; Stigneev: Vek fotografii, S. 56-65, 78-111; Irina Tchmyreva: The History of Russian Photography, in: Václav Macek (Hrsg.): The History of European Photography, Bd. 1. 1900-1938, Bratislava 2010, S. 509-553; Margarita Tupitsyn: The Soviet Photograph 1912-1937, Yale 1996.

72 Rosalinde Sartorti: Pressefotografie und Industrialisierung in der Sowjetunion. Die Pravda 1925-1933, Berlin 1981; Christopher Stolarski: The Rise of Photojournalism in Russia and the Soviet Union, 1900-1931, Baltimore, Univ., Diss., 2013; Erika Wolf: When Photographs Speak, to Whom Do They Talk? The Origins and Audience of SSSR na Stroike (USSR in Construction), in: Left History, Jg. 6/200o, Heft 2, S. 53-82.

73 Elena Valentinovna Barchatova: Russkaja svetopis'. Pervyj vek fotoiskusstva, 1839-1914, Sankt Peterburg 2009; Anatolij Petrovič Popov: Iz istorii rossijskoj fotografii, Moskva 2010; Stigneev: Vek fotografii.

74 Tat'jana Nikolaevna Šipova: Fotografy Moskvy - na pamjat' buduščemu, 1839-1930. Al'bom-spravočnik, Moskva 2001; Tat'jana Nikolaevna Šipova: Moskovskie fotografy 18391930. Istorijam sokovskoj fotografii, Moskva 2012; o. A.: Fotogtafii na pamjat'. Fotografy Nevskogo prospekta 1850-1950, Sankt Peterburg 2003; Sergej Golota u. a.: Fotografy 
Technikgeschichte auseinander. ${ }^{75}$ Die soziale Bedeutung des Bildmediums ist in der Forschung weitgehend unterbelichtet geblieben. Es liegen kaum historische Forschungen über den Einfluss der Fotografie auf soziale Praktiken und den Alltag der Menschen im Zarenreich vor oder darüber, welche gesellschaftlichen und wirtschaftlichen Strukturen sich durch die Fotografie veränderten. ${ }^{76}$ Die vorliegende Arbeit widmet sich diesem Forschungsdesiderat und untersucht die Bedeutung des neuen Bildmediums in der Industrie des Zarenreichs.

Darüber hinaus werden die gesellschaftlichen Bilddiskurse über Industrialisierung untersucht. Es wird analysiert, wie Akteure in der zweiten Hälfte des 19. und zu Beginn des 20. Jahrhunderts versuchten, mittels der Fotografie Einfluss auf die Wahrnehmung von Fabriken und Industrialisierung zu nehmen beziehungsweise neue Bilder einer sauberen, wohlgeordneten russischen Modernisierung zu entwerfen. Je nach Verwendungskontext fungierten die Fabrikfotografien als Repräsentationen und Visualisierungen einer positiven Sicht auf die Industrie. ${ }^{77}$ Eine Sichtweise, die insbesondere Industrielle zu verbreiten suchten. Bildinhalte, Ästhetik und Verwendung der Aufnahmen werden ebenso analysiert wie die Kommunikation zwischen Unternehmern und Fotografen sowie die Bedeutung, die Aufträge für Industriefotografien für Lichtbildner hatten.

Unter dem Begriff Wahrnehmung wird in dieser Arbeit nicht allein die Aufnahme der visuellen Informationen über Fotografien verstanden. Zum Prozess der Wahrnehmung werden auch die Verarbeitung dieser Informationen und ihr Einfluss auf das Handeln der Akteure und deren Verständnis der Realität gezählt. ${ }^{78}$

Char'kovskoj gubernii. 1851-1917. Chudožestvenno-istoričesij fotoal'bom spravočnobiografičeskogo charaktera, Char'kov 2008.

75 Renner: Der Visual Turn und die Geschichte der Fotografie im Zarenreich, S. 412-413. Dies ist keine Besonderheit der russischen Fotografieforschung. Auch in Westeuropa und den USA dominieren häufig gerade diese Aspekte die wissenschaftlichen Untersuchungen. Jens Jäger: Fotografiegeschichte(n). Stand und Tendenzen der historischen Forschung, in: Archiv für Sozialgeschichte, Jg. 48/2008, S. 511-537, S. 515 .

76 Interessanterweise ist die Untersuchung des Entstehungskontextes von Werken eine Grundlage der Kunstgeschichte. Für die Fotografie hat sich diese Fragestellung jedoch eher weniger durchgesetzt. Bernd Roeck: Visual turn? Kulturgeschichte und Bilder, in: Geschichte und Gesellschaft, Jg. 29/2003, Heft 2, S. 294-315, S. 302-303.

77 Zur hier verwendeten Definition von Repräsentation: Jörg Baberowski: Selbstbilder und Fremdbilder. Repräsentation sozialer Ordnung im Wandel, in: ders.; Hartmut Kaelble; Jürgen Schriewer (Hrsg.): Selbstbilder und Fremdbilder. Repräsentation sozialer Ordnung im Wandel, Frankfurt a. M., New York 2008, S. 9-13, S. 11-12.

78 Hierzu auch: Ulrich Ansorge; Helmut Leder: Wahrnehmung und Aufmerksamkeit, Wiesbaden ${ }^{2} 2017$, S. 5-6; Eva Schürmann: Sehen als Praxis. Ethisch-ästhetische Studien zum Verhältnis von Sicht und Einsicht, Frankfurt a. M. 2008, S. 144-146. 
Die Konzentration auf Fotografien aus Fabrikkontexten ergänzt die Forschungen der osteuropäischen Geschichte und der Fotografiegeschichte zu einem Themenfeld, das nicht nur im russischen Kontext bislang wenig Aufmerksamkeit fand. Zur Industriemalerei liegen einige Studien für Deutschland, England und Frankreich vor. ${ }^{79}$ Erstaunlich wenige Arbeiten existieren hingegen zu Industrie- und Fabrikfotografien aus dem 19. und frühen 20. Jahrhundert. Lange waren Industriefotografien nicht als Teil der Kunstfotografie anerkannt und gehörten damit nicht zum klassischen Forschungsobjekt der Kunstgeschichte. ${ }^{80}$ Erst Ende der 1970er und während der 1980er Jahre entstanden erste Arbeiten, die sich näher mit Fotografien aus dem Bereich der Industrie beschäftigten. ${ }^{81}$

In einer Phase, in der sich die Wirtschaftsstrukturen in Westeuropa und den USA stark veränderten und die klassische Industriemoderne vielerorts verschwand, ${ }^{82}$ faszinierten fotografische Abbildungen vergangener Produktionsabläufe und Arbeitsformen zunehmend mehr Wissenschaftler. ${ }^{83}$ Viele Studien zur Industriefotografie beschränkten sich auf Fotografien aus einer Region oder einem Firmennachlass. ${ }^{84}$ Andere Autoren konzentrierten sich auf ein

79 Zu Deutschland: Paul Brandt: Schaffende Arbeit und Bildende Kunst, Bd. 2. Vom Mittelalter bis zur Gegenwart, Leipzig 1928; Klaus Türk: Bilder der Arbeit. Eine ikonografische Antologie, Wiesbaden 2000; Ferdinand Ullrich: Zur Darstellung industrieller Wirklichkeit in der Malerei, in: Industriebilder aus Westfalen. Gemälde, Aquarelle, Handzeichnungen, Druckgrafik 1800-1960. Westfälisches Landesmuseum für Kunst und Kulturgeschichte Münster Landschaftsverband Westfalen-Lippe, 7.9. bis 21.10.1979, Münster 1979, S. 19-33. Zu England: Francis Donald Klingender: Art and the Industrial Revolution, London 1947. Zu Frankreich: Nicolas Pierrot: Les images de l'industrie en France. Peintures, dessins, estampes 1760-1870, Paris, Univ., Diss., 2010.

8o Ähnlich Überlegungen formuliert auch: Pugh: Industrial Image, S. 9.

81 Forrest Jack Hurley (Hrsg.): Industry and the Photographic Image. 153 Great Prints from 1850 to the Present, New York 1980; o. A.: Le Grand Oeuvre. Photographies des grands travaux, 1860-1900, Paris 1983; André Rouillé: Les images photographiques du monde du travail sous le Second Empire, in: Actes de la recherche en sciences sociales, Jg. 14/1984, Heft 54, S. 31-43; Matz: Industriefotografie.

$82 \quad$ Zu dieser Thematik: Manuel Castells: Der Aufstieg der Netzwerkgesellschaft, Bd. 1. Das Informationszeitalter, Opladen 2001, S. 237-245; Anselm Doering-Manteuffel; Lutz Raphael: Nach dem Boom. Perspektiven auf die Zeitgeschichte seit 1970, Göttingen 2008.

83 Diese Faszination steht in Zusammenhang mit dem Geschichtsboom der 1970er und 1980er Jahre. Siehe hierzu: Kapitel „Inszenierte Geschichte - Firmenjubiläen“, S. 179-240.

84 Klaus Tenfelde (Hrsg.): Bilder von Krupp. Fotografie und Geschichte im Industriezeitalter, München 1994; Liselotte Kugler (Hrsg.): Die AEG im Bild, Berlin 2000; Henning Rogge: Fabrikwelt um die Jahrhundertwende am Beispiel der AEG Maschinenfabrik in Berlin-Wedding, Köln 1983; Lisa Kosok; Stefan Rahner (Hrsg.): Industrie und Fotografie. Sammlungen in Hamburger Unternehmensarchiven, Hamburg 1999; David E. Nye: Image Worlds. Corporate Identities at General Electric, 1890-1930, Cambridge, London 1985; Auch aktuell aus Österreich: Martin Keckeis: Fotografien im Dienste expansiver Unternehmensstrategien 1855-1879. Die k. k. Staats-Eisenbahn-Gesellschaft zwischen Paris und 
Medium und untersuchten die Fotografien jeweils in einem entsprechenden medienspezifischen Kontext. ${ }^{85}$ Die vorliegende Arbeit widmet sich im Gegensatz dazu Industriefotografien in unterschiedlichen Medien und ermöglicht dadurch einen differenzierteren Blick auf den Forschungsgegenstand.

Während frühe Industriefotografien in Frankreich in den letzten Jahren ein beliebtes Forschungsthema waren, sind sie in der osteuropäischen Geschichte kaum präsent. Margarita Sergeevna Štiglic gehört zu den wenigen Wissenschaftlern, die sich mit vorrevolutionären Fotografien russischer Fabriken beschäftigen. Als Industriearchäologin nutzt sie die Abzüge jedoch ausschließlich, um materialtechnische und architektonische Neuentwicklungen in der Industriearchitektur St. Petersburgs herauszuarbeiten und geht weder auf den Entstehungskontext der Bilder noch auf ihre ästhetischen Besonderheiten ein. ${ }^{86}$ Die vorliegende Arbeit nutzt die bestehenden Forschungen zu Westeuropa und den USA, um die Ergebnisse der Untersuchung zur russischen Fabrikfotografie in einen internationalen Kontext zu stellen. Zudem trägt sie dazu bei, Bilder russischer Fabriken als lohnenden Untersuchungsgegenstand für Analysen der Industrie- und visuellen Kultur zu etablieren. ${ }^{87}$

\subsection{Industriefotografie in unterschiedlicher Rahmung: Themen und Kapitel}

Die Arbeit folgt dem Motiv der Fabrik durch die Bildwelten des Zarenreichs. Dies legt eine Gliederung nach Medien - Fotoalben, Festschriften, illustrierten

dem Banat, in: Monika Faber; ders. (Hrsg.): Bildpolitik der Ingenieure. Fotokampagnen der k. k. privilegierten österreichischen Staats-Eisenbahn-Gesellschaft 1855-1879, Salzburg 2016, S. 19-66.

85 So die Beiträge im folgenden Sammelband: Denis Woronoff, (Hrsg.): Les images de l'industrie de 1850 à nos jours. Actes du colloque tenu à Bercy, les 28 et 29 juin 2001, Paris 2002. Die folgenden Arbeiten konzentrieren sich auf fotografische Abzüge und Fotoalben: Assegond: La photographie du travail; Alf Lüdtke: Industriebilder - Bilder der Industriearbeit? Industrie und Arbeitsphotographie von der Jahrhundertwende bis in die 1930er Jahre, in: Historische Anthropologie, Jg. 1/1993, Heft 3, S. 394-430; Iris Metje: Harmonische Einheit und Spuren des Wandels. Industrielandschaft als fotografische Landschaft, in: Fotogeschichte. Beiträge zur Geschichte und Ästhetik der Fotografie, Jg. 31/2011, Heft 120, S. $49-56$.

86 Margarita Štiglic: Promyšlennaja architektura Peterburga v sfere „industrial'noj archeologii“, Sankt-Peterburg 2003.

87 Sowjetische Industriefotografien sind im Gegensatz zu ihren Vorgängern durchaus in der Forschung präsent. Erika Wolf: The Soviet Union. From Worker to Proletarian Photography, in: Jorge Ribalta (Hrsg.): The Worker Photography Movement [1926-1939]. Essays and Documents, Madrid 2011, S. 32-46, Stigneev: Vek fotografii. 
Zeitschriften und Postkarten - nahe. Durch alle Kapitel zieht sich die Frage nach neu entstandenen Verwendungsweisen der aufgenommenen Bilder sowie das Verhältnis von Mensch und Maschine in den Fotografien. Thematisiert wird, welche Funktionen Menschen auf fotografischen Aufnahmen in unterschiedlichen Kontexten erfüllten, wer einen Platz auf den Bildern erhielt und welche Machtverhältnisse die visuellen Inszenierungen widerspiegeln.

Den Auftakt bilden zwei Kapitel, die sich mit dem historischen Kontext der Fotografie auseinandersetzen. Hier spielt die direkte Quellenanalyse zunächst eine untergeordnete Rolle. Vielmehr widmet sich das erste Kapitel der Entwicklung der russischen Industrie und Fotografie. Beide Prozesse werden parallel verfolgt, wodurch die Fotografie als Gewerbe einen Platz innerhalb des Wirtschaftslebens im Zarenreich erhält. Weiter gibt das Kapitel einen kurzen Überblick der zeitgenössischen Diskussionen innerhalb der russischen Elite mit Blick auf die Frage, welchen Weg die russische Wirtschaft einschlagen solle und welches Wirtschaftssystem den nationalen Besonderheiten Russlands am besten entspräche. Im anschließenden Kapitel werden die visuellen Vorgänger der Industriefotografien untersucht. Im Fokus stehen einerseits Werke aus der Malerei, andererseits Stiche und Lithografien, die Fabrikansichten zeigen. In der Analyse wird herausgearbeitet, welche Traditionen der Industriemalerei sich in Westeuropa ausbildeten, inwiefern sich Künstler im Zarenreich an diesen Vorbildern orientierten, aber auch wie sie sich abgrenzten. Das Verhältnis zwischen Fotografie und Malerei blieb bis zum Ersten Weltkrieg sehr eng, so dass der Überblick vom 18. bis ins frühe 20. Jahrhundert reicht.

Einen ersten Blick hinter die Eingangstore russischer Fabriken erhält der Leser in einem Überblickskapitel zum Verhältnis von Fotografie und Fabriken. Hier wird herausgearbeitet, welche Funktionen die neuen Bilder innerhalb der Unternehmen übernahmen. Weiter zeigt die Studie, welche neuen Praktiken sich mit der Verbreitung fotografischer Aufnahmen ausbildeten und wie wichtig das Image dieses fortschrittlichen Bildmediums gerade in einem industriellen Kontext war.

Den Hauptteil der Arbeit bilden fünf Quellenkapitel, von denen vier nach den in ihnen analysierten Träger-Medien bezeichnet sind. Das fünfte Quellenkapitel widmet sich der Übergangsperiode des Ersten Weltkriegs. Das erste Quellenkapitel beschäftigt sich mit Fotoalben. Hier stehen neben der Verwendung von Fotografien besonders die Motive der Aufnahmen im Fokus. Die Bildanalyse in diesem Kapitel arbeitet die visuellen Spezifika von Industriefotografien heraus und stellt exemplarisch ihre Charakteristika vor. Das Kapitel geht der Frage nach, wie Lichtbildner Fabriken als Orte inszenierten und welche virtuellen Räume sie mit ihren Bildern schufen. Angeordnet in Alben fügten sich die Aufnahmen zu Bildnarrativen, die den Blick der Betrachter auf 
ein Unternehmen, dessen Werkshallen und Beschäftigte leiteten. Es lässt sich beobachten, welche Aspekte des Arbeitslebens Teil der visuellen Präsentation waren und welche Bereiche keinen Platz zwischen den Albendeckeln fanden. Die Bildanalyse wird in diesem Kapitel um eine Objektanalyse erweitert, die die Ausgestaltung von Alben in die Interpretation einbezieht und an einem Beispiel den Bedeutungswandel einzelner Fotografien verdeutlicht.

Das zweite Quellenkapitel lenkt den Blick auf eine spezifische Form der Firmenpublikation - auf die Festschrift und auf die im langen 19. Jahrhundert immer beliebter werdende Tradition des Firmenjubiläums. Anlässlich dieser Jahrestage traten Unternehmer in einer breiten gesellschaftlichen Öffentlichkeit in Erscheinung. Untersucht wird einerseits ihre Rolle als Geschichtsschaffende, andererseits die von ihnen angewandten Strategien, mit denen sie eine virtuelle Erinnerungsgemeinschaft bilden wollten, in deren Zentrum die eigene Fabrik und deren Geschichte stand.

Das starke Wirtschaftswachstum der 189oer Jahre und die zunehmende industrielle Produktion hatten zur Folge, dass der Massenkonsum im Zarenreich an Bedeutung gewann. Teil dieser Entwicklung waren illustrierte Zeitschriften - diese bilden die Grundlage des dritten Quellenkapitels. Es untersucht, ob Redakteure Fotografien von Fabriken und Industrieanlagen als geeignet ansahen, um mit ihnen ihre Leserschaft zu unterhalten, oder welche Bedingungen erfüllt sein mussten, damit die Aufnahmen einen Weg in die beliebten Blätter fanden. Außerdem zeigt die Analyse von sechs russischen Zeitschriften, in welchem Zusammenhang Fotografien die Schattenseite der Industrialisierung thematisierten. ${ }^{88}$

Die Untersuchung des vierten Quellenkapitels richtet ihr Augenmerk auf die Unterschiede zwischen Provinz und imperialen Zentren. Am Beispiel von Bildpostkarten verfolgt das Kapitel, in welchen Regionen des Russischen Reichs Fabrikillustrationen zum Bildrepertoire gehörten, und formuliert Thesen, warum sich die Präsenz von Fabriken auf Korrespondenzkarten je nach Ort stark unterschied.

Der Erste Weltkrieg stellte für die russische Industriefotografie einen wichtigen Einschnitt dar. Aus diesem Grund weicht das letzte Quellenkapitel von der nach Medien geordneten Gliederung ab. Es verfolgt stattdessen, wie sich das Image der russischen Industrie vor dem Hintergrund des militärischen

88 Im Zarenreich gab es keine prominenten Zeitgenossen, die die Lebens- und Arbeitsbedingungen der Fabrikarbeiter und städtischen Unterschichten fotografisch dokumentierten, um Gesetzesänderungen oder staatliche Hilfe zu fordern. Das Vorgehen des amerikanischen Fotografen Lewis E. Hine (1874-1940), der sich mit seinen sozialkritischen Aufnahmen von Kindern in Fabriken für eine gesetzliche Regelung der Kinderarbeit in den USA einsetzte, hat in Russland kein Pendant. 
Konflikts veränderte. Zum einen skizziert das Kapitel, das neue Interesse, das Fabrikfotografien bei Redakteuren illustrierter Zeitschriften hervorriefen. Dabei rückt der internationale Aspekt des Themas ebenso in den Fokus wie eine neue Bildästhetik, die russische Fotografen vor dem Hintergrund des Krieges für sich entdeckten. Zum anderen werden Fotografien von Firmenbesuchen des Zaren Nikolaus II. (1868-1918) untersucht, der im Ersten Weltkrieg mehrfach Rüstungsunternehmen besichtigte.

Der Ausblick gibt anhand von vier kurzen Fallbeispielen einen Einblick, wie sich russische Fabrikfotografien in Fotoalben, Festschriften, illustrierten Zeitschriften und auf Postkarten nach der Oktoberrevolution entwickelten. Er skizziert, wodurch sich die sowjetischen Bilder von ihren Vorgängern aus dem Zarenreich unterschieden, und zeigt auf, in welchen Bereichen Narrative oder Elemente der Bildsprache über den politischen Umbruch von 1917 hinaus Bestand hatten.

Wie eigneten sich Menschen in der Vergangenheit ihre Wirklichkeit an? Für diese zentrale Frage der kulturwissenschaftlichen Forschung spielten in der zweiten Hälfte des 19. und im frühen 20. Jahrhundert Bilder eine entscheidende Rolle. Immer wieder betonen Autoren in der Forschungsliteratur, dass sich Bilder stärker einprägen als Worte. Hinzu kommt das besondere Gefühl der "Augenzeugenschaft", das Bilder ihren Betrachtern vermitteln können. ${ }^{89}$ Dies gilt für die Fotografie in besonderem Maße. Fotografien ließen sich vergleichsweise einfach transportieren und vermittelten ihrem Publikum einen Einblick in Räume, die für sie nicht immer zugänglich waren. Insbesondere Fabrikanten machten sich diese Eigenschaften zunutze. Sie prägten dadurch das Bild, das sich ihr Publikum von Fabrikanlagen machte, die es nicht selbst betreten konnte. Neben den Industriellen verbreiteten Fotografen, Redakteure und Herausgeber von Postkarten Bilder der russischen Industrie und nahmen ihrerseits darauf Einfluss, wie die Menschen Fabriken wahrnahmen. Die Arbeit rückt Industrie, Fabriken und Fotografie in den Fokus der Analyse. Sie bereichert auf diese Weise Forschungen zur russischen Industrialisierung um kulturwissenschaftliche Komponenten und etabliert fotografische Quellen als eigenständige Quellen erster Ordnung für die Geschichte des Zarenreichs.

89 Burke: Augenzeugenschaft; Marion G. Müller: Grundlagen der visuellen Kommunikation. Theorieansätze und Methoden, Konstanz 2003, S. 88. 